\title{
Feature Extraction Based Estimation of Rain Fall By Cross Correlating Cloud RADAR Data
}

\author{
Shaik Basha ${ }^{1 *}$, K. Sridhar Reddy ${ }^{1}$, J.Krishna Chaithanya ${ }^{1}$, B.U.V Prashanth ${ }^{2}$ \\ ${ }^{1}$ Associate Professor, ${ }^{2}$ Assistant Professor Dept. of ECE, Vardhaman College of Engineering. Hyderabad, \\ Andhra Pradesh, India.
}

\begin{abstract}
In this paper we present the feature extraction based estimation of rain fall by cross correlating cloud RADAR Data. The idea is to select a square box of around $200 \times 200$ pixels around the point of interest and take the cross correlation between the last picture and one that is 5 or 10 minutes older. We then determine the wind direction and speed by finding the highest point in the correlation. Last step is to interpolate the data acquired in a tagged format to the latest data in the up-wind direction to get a prediction for the near future. The basic principle works, but it is hard to get a good estimate of the wind direction.
\end{abstract}

Keywords - Feature Extraction, Cross correlation, Rain Fall, RADAR, Image Processing.

\section{INTRODUCTION}

In this paper we predict the amount of rainfall by analyzing data from the moving cloud data captured by radar. This information used to be available with 15 minutes interval and delayed by 2 hours. The moving cloud pattern is a transparent avi file drawn over the map, so the data is easily extracted. We determine the structure in the clouds to get a decent prediction, but sometimes we also get large errors even while the cloud movement is very obvious. The simulations were carried out with correlating random noise, and here we subtract the mean value obtained, otherwise the peak corresponding to the proper shift is overshadowed by a central diamond shaped peak that is caused by the correlation of the D.C term.

\section{ALGORITHM DESIGN}

The algorithm is designed according to the following steps:-

1. Start

2. Read the .avi (Video file) captured by Cloud RADAR.

3. Initialize the dark cloud value to 50 .

4. Perform RGB to Gray conversion to do morphological processing.

5. Compute the Cloud traffic object.

6. Remove dark clouds.

7. Remove the markings and other non-disk shaped structures.

8. Remove small structures.

9. Get the area and centroid of each remaining object in the frame.

10. Find the object with the largest area is the light-colored cloud.

11. Create a copy of the original frame and tag the cloud by changing the centroid pixel value to red.

12. Calculate the frame rate

13. Play the tagged clouds and frame rate combined together.

The MATLAB script is designed according to the above algorithm is as illustrated below.

CloudtrafficObj $=$ mmreader('clouds.avi')

implay('clouds.avi');

darkCloudValue $=50$;

darkCloud $=$ rgb2gray $(\operatorname{read}($ CloudtrafficObj, 71$))$;

noDarkCloud = imextendedmax (darkCloud, darkCloudValue);

imshow(darkCloud)

figure, imshow(noDarkCloud)

sedisk $=$ strel('disk',2);

noSmallStructures = imopen(noDarkCloud, sedisk);

imshow(noSmallStructures)

nframes = get(CloudtrafficObj, 'NumberOfFrames');

$\mathrm{I}=\operatorname{read}($ CloudtrafficObj, 1$)$;

taggedClouds= zeros([size(I,1) size(I,2) 3 nframes], class(I)); 
for $\mathrm{k}=1:$ nframes

singleFrame $=\operatorname{read}($ CloudtrafficObj, $\mathrm{k})$;

I = rgb2gray(singleFrame);

noDarkClouds = imextendedmax (I, darkCloudValue);

noSmallStructures = imopen (noDarkClouds, sedisk);

noSmallStructures $=$ bwareaopen (noSmallStructures, 150$)$;

taggedClouds $(:,:, ;, \mathrm{k})=$ singleFrame;

stats = regionprops(noSmallStructures, $\{$ 'Centroid','Area' $\})$;

if $\sim$ isempty ([stats.Area])

areaArray $=[$ stats.Area $]$

[junk,idx] = $\max ($ areaArray $)$;

$\mathrm{c}=\operatorname{stats}(\mathrm{idx})$.Centroid;

$\mathrm{c}=$ floor(fliplr(c))

width $=2$;

row $=\mathrm{c}(1)$-width:c(1)+width;

col = c(2)-width:c(2)+width;

taggedClouds $($ row $, \mathrm{col}, 1, \mathrm{k})=255$;

taggedClouds(row,col, $2, \mathrm{k})=0$;

taggedClouds $($ row $, \mathrm{col}, 3, \mathrm{k})=0$;

endend

frameRate $=$ get $($ CloudtrafficObj, 'FrameRate' $)$;

implay(taggedClouds, frameRate);

\section{FLOW CHART DESIGN}

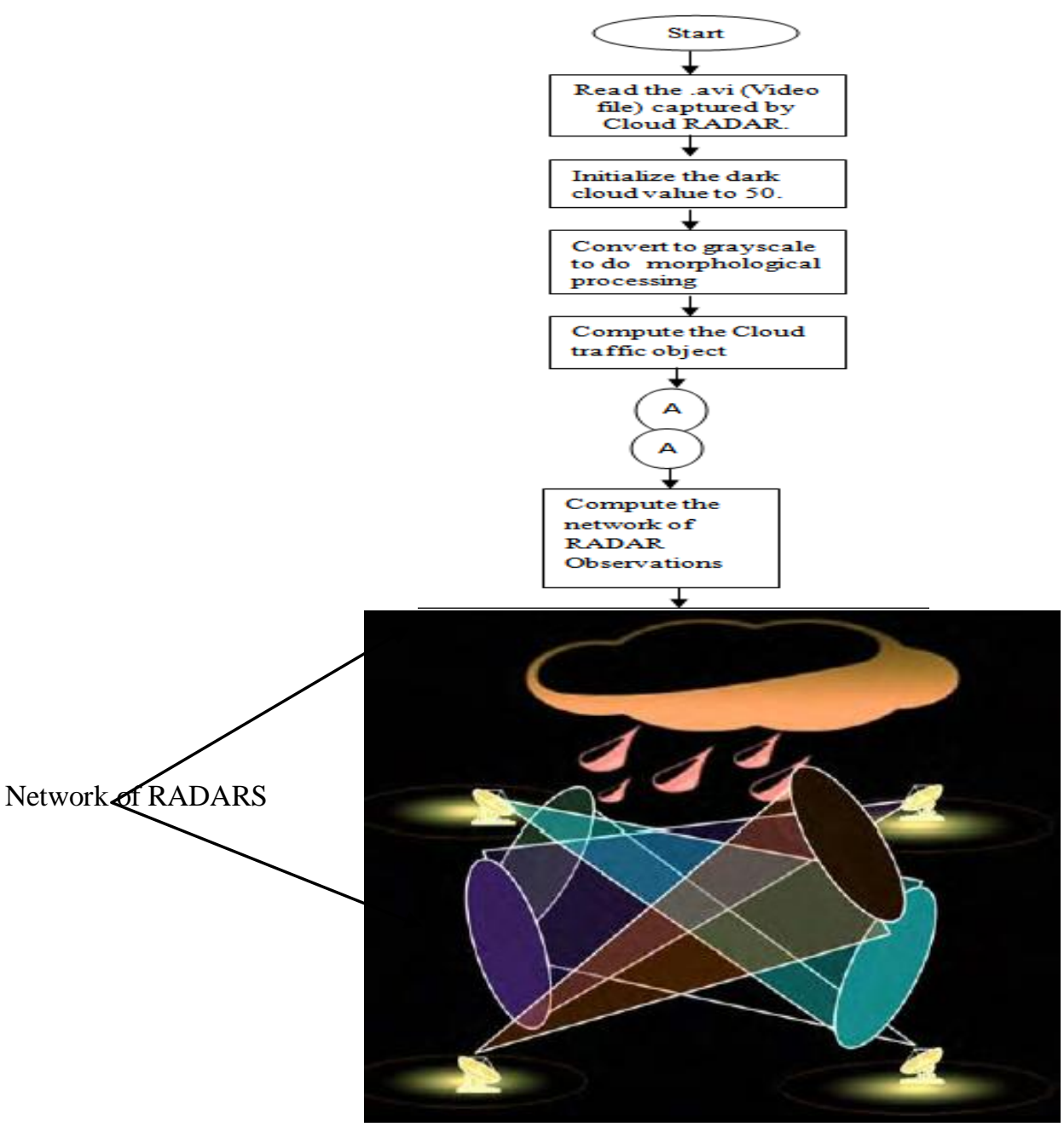




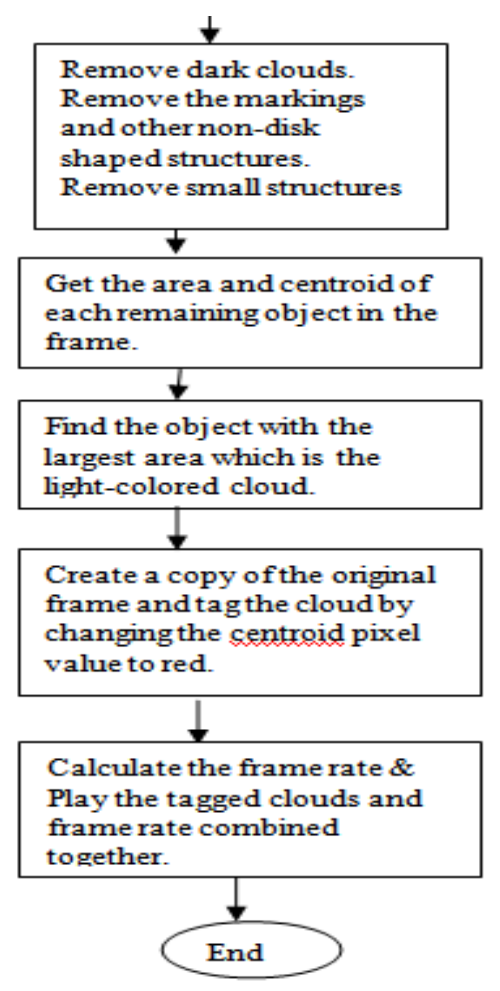

\section{UTPUT RESULTS}

We have obtained the following output results as illustrated (in Fig 1,2,3,4) in MATLAB R2012-a real time simulation environment:-

Video Parameters:-29.92 frames per secondRGB value as 24 540x360

Total video frames available $=1208$

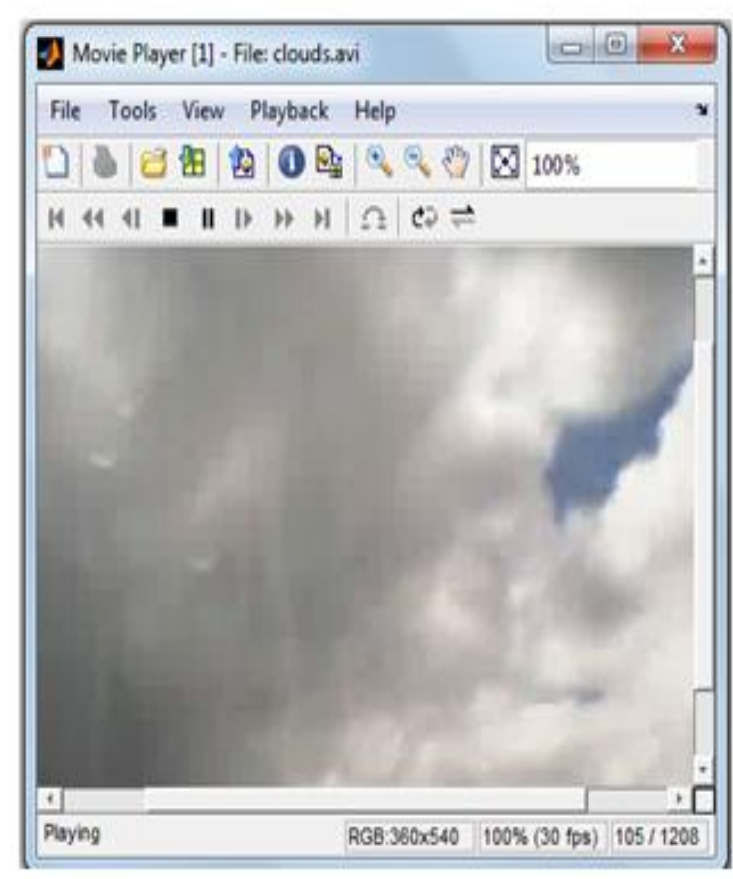

Figure 1 .avi (Video file) captured by Cloud RADAR.

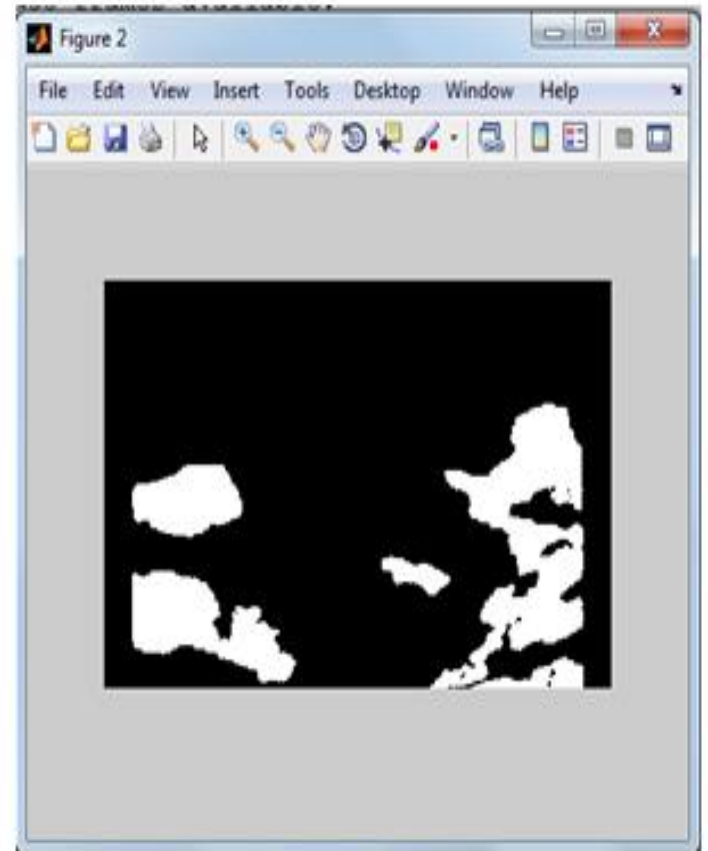

Figure 2 Morphological Processing 


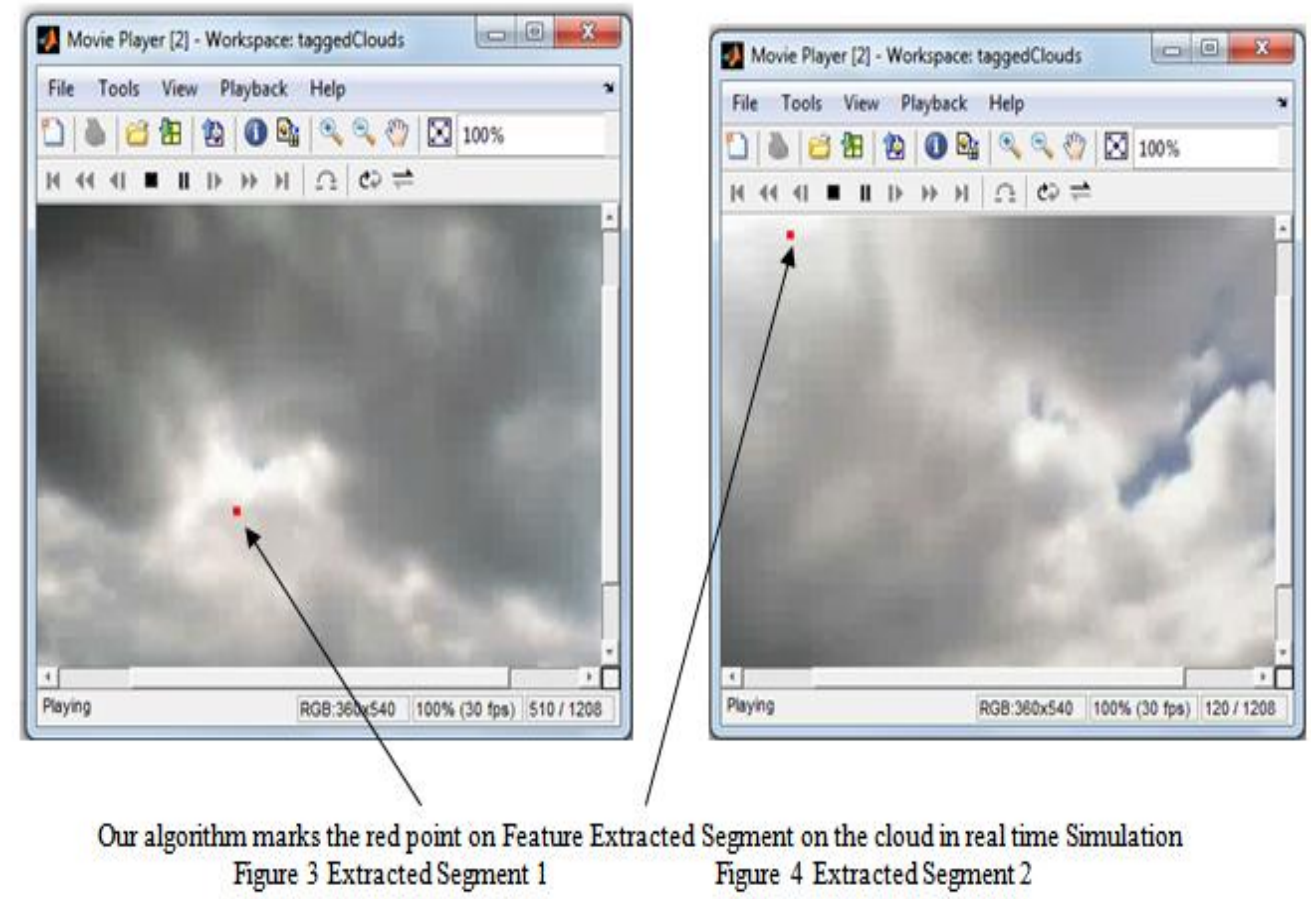

The red point on the feature extracted segment on the cloud indicates the color of the cloud as dark colored. If the color is dark which is indicated by the red point as seen in figures 3 and 4, then we can predict the rainfall at that instant of time.

\section{CONCLUSION}

Here we presented the Feature Extraction Based Estimation of Rain Fall By Cross Correlating Cloud RADAR Data. We selected a square box of around 200x200 pixels around the point of interest and take the cross correlation between the last picture and one that is 5 or 10 minutes older. We then determined the color of the cloud by finding the highest point in the correlation. Finally we interpolated the data acquired in a tagged format to the latest data predicting the color of the cloud to get a prediction for the near future.

\section{REFERENCES}

[1]. J. Xu, "Contented Based Image Retrieval System Design and Implementation," 2004.

[2]. S. H. Yanbing LI, Yuanxiang Li, Jingqiu Zhai, "CBIR methods in satellite cloud image retrieval," JOURNAL OF PLA UNIVERSITY OF SCIENCE AND TECHNOLOGY(NATURAL SCIENCE EDITION), vol. 9, pp. 130-136, 2008. (Pubitemid 351766028)

[3]. M. Heidt, T. Dörnemann, K. Dörnemann, and B. Freisleben, "Omnivore: Integration of Grid Meta-Scheduling and Peer-to-Peer Technologies," in 2008 Eighth IEEE International Symposium on Cluster Computing and the Grid (CCGRID), 2008, pp. 316-323.

[4]. Foster and C. Kesselman, The grid: blueprint for a new computing infrastructure. San Francisco, CA, USA: Morgan Kaufmann, 2004.

[5]. F. Dabek, B. Zhao, P. Druschel, J. Kubiatowicz, and I. Stoica, "Towards a common API for structured peer-to-peer overlays" in 2nd International Workshop on Peer-to-Peer Systems (IPTPS' 03), 2003, pp. 33-44.

[6]. S. Saroiu, K. Gummadi, R. Dunn, S. Gribble, and H. Levy, "An analysis of internet content delivery systems," in Proceedings of the 5th USENIX Symposium on Operating Systems Design and Implementation (OSDI '02), Berkeley, CA, USA, 2002, pp. 315-327.

[7]. Arking and J. D. Childs, "Retrieval of Cloud Cover Parameters from Multispectral Satellite Images," Journal of Climate and Applied Meteorology, vol. 24, pp. 322-333, 1985.

[8]. Herbreteau, G. Salem, M. Souris, J. Hugot, and J. Gonzalez, "Thirty years of use and improvement of remote sensing, applied to epidemiology: From early promises to lasting frustration," Health \& Place, vol. 13, pp. 400-403, 2007. (Pubitemid 46157014) 